\title{
Operant methods for mouse psychoacoustics
}

\author{
KARIN B. KLINK, GARNET BENDIG, and GEORG M. KLUMP \\ Carl von Ossietzky Universität Oldenburg, Oldenburg, Germany
}

\begin{abstract}
Tone detection thresholds for a $10-\mathrm{kHz}$ tone in NMRI mice were determined in psychoacoustic experiments using both a constant-stimuli procedure and a two-down/one-up adaptive-tracking procedure in the same subjects and applying identical threshold criteria (70.7\% response probability). Constant-stimuli thresholds were on average $24 \mathrm{~dB}$ lower than adaptive-tracking thresholds, and there was a trend indicating that constant-stimuli thresholds were less variable than adaptive-tracking thresholds. Furthermore, in the constant-stimuli procedure the number of trials constituting the psychometric function could be reduced from 100 to 50 trials without a large loss of accuracy of threshold determination. In the constant-stimuli procedure, the threshold value was affected by the threshold criteria. The lowest and least variable constant-stimuli thresholds were obtained by applying signal detection theory and a criterion of $d^{\prime}=1$. Thus, the constant-stimuli procedure in combination with signal detection theory appears to be better suited than the adaptive-tracking procedure to determine auditory sensory thresholds.
\end{abstract}

Various laboratory strains of the house mouse (Mus musculus) have developed into model organisms for the study of auditory processing (see, e.g., Willott et al., 2003; Zheng, Johnson, \& Erway, 1999), and the high availability of mutant and genetically engineered mouse strains, in combination with knowledge of the complete mouse genome (Waterston et al., 2002), offers an unprecedented possibility to study the genetic basis of the mechanisms underlying hearing. Standard procedures for studying auditory function in mammals involve both the recording of neural responses at various levels of the auditory system and psychoacoustic methods that have been adapted to the comparative study of hearing. In the mouse, behavioral studies of auditory function employ a variety of methods that range from exploiting basic reflexes and their modification (for startle response and prepulse inhibition, see, e.g., Ison \& Agrawal, 1998; Willott et al., 2003; for the Preyer reflex, Schleidt, 1952; for galvanic skin response, Berlin, 1963) to operant tasks that are adapted to emulate psychoacoustic procedures used in humans (e.g., Heffner \& Masterton, 1980; Markl \& Ehret, 1973; Prosen, Bath, Vetter, \& May, 2000; for a review of methods with the mouse, see Heffner \& Heffner, 2001). With respect to the presentation of stimuli, two different schemes are commonly used in psychoacoustic studies: adaptive-tracking

This study was funded by a grant from the Deutsche Forschungsgemeinschaft to researchers in Collaborative Research Center SFB 517. The care and treatment of the animals was approved by the Bezirksregierung Weser-Ems (permit 509.8-42502-35/7) and followed the NIH Guide for the Care and Use of Laboratory Animals. Günter Ehret provided the mice for this study. We thank J. Ison and P. Allan for discussion. Correspondence relating to this article may be sent to G. M. Klump, Carl von Ossietzky Universität Oldenburg, IBU, Fak. 5, AG Zoophysiologie \& Verhalten, Postfach 2503, 26111 Oldenburg, Germany (e-mail: georg.klump@uni-oldenburg.de). procedures, in which the value of the stimulus parameter depends on the subject's previous response; and constantstimuli procedures, in which the values of the stimulus parameters are randomly drawn from a predetermined set. In studies of human psychoacoustics, adaptive-tracking procedures are often preferred, because they are considered especially efficient for collecting data close to the sensory threshold and require no previous knowledge of the approximate threshold value (e.g., Dai, 1995; Leek, 2001; Leek, Hanna, \& Marshall, 1992; Watson \& Fitzhugh, 1990). In contrast, constant-stimuli procedures are often applied in psychophysical experiments with animal subjects, because experimenters assume that these procedures provide better means to keep the subject under "stimulus control" - that is, to assure a tight link between the presented stimulus and the response. This is thought to be achieved in the constant-stimuli procedure by the frequent presentation of stimuli well above threshold throughout experimental sessions, whereas in the adaptive-tracking procedure the subject would be presented with more stimuli close to threshold (e.g., Dai, 1995; Niemiec \& Moody, 1995). In this study, we compare data obtained with both procedures in the same subjects and test the suitability of these procedures for studying mouse psychoacoustics.

\section{METHOD}

\section{Subjects}

The animals used in this experiment were adult house mice (Mus musculus), 4 females and 2 males, of the NMRI strain bred by Günter Ehret (Ulm, Germany), who obtained the initial breeding stock from Merck, Darmstadt (Ehret, personal communication). The NMRI strain is an outbred mouse strain that was kept at the US Naval Medical Research Institute (hence, the name NMRI mouse). The NMRI mouse is a normal-hearing albino that is commonly used as a control in experiments.

The subjects' age ranged between 3 and 8 months during the period of testing. They were housed in individual cages (Eurostandard 
Type III H, $43 \times 27 \times 19 \mathrm{~cm}$; Tecniplast) with a hiding possibility ("mouse house," Tecniplast) and a layer of wood shavings as bedding material (Raiffeisen). All cages were stored in a ventilated cage rack (Slim Line Sealsafe, Tecniplast). The animals were kept at a weight of between 22 and $36 \mathrm{~g}$ and had unrestricted access to water. The food rewards during the experiments consisted of 20-mg pellets (BioServe Dustless Precision pellets, Formula FO163), and additional rodent pellets (Altromin Type 1314) were given after the experiments to keep the animals' weights about constant. The animals were moved from their cages to the experimental cage using a small transfer cage.

\begin{abstract}
Apparatus
The animals were tested in a sound-attenuating chamber (Industrial Acoustics IAC $403 \mathrm{~A}$, inside dimensions $245 \times 227 \times 240 \mathrm{~cm}$ ) lined with two to three layers of sound-absorbing panels (either Waffle Type 70/125 wedges mounted on one layer of Plano Type 50/0 SF or Illsonic Pyramide 100/100 wedges mounted on two layers of Plano; illbruck acoustic). The wedges had an absorption coefficient of more than .99 for frequencies above $500 \mathrm{~Hz}$. The doughnutshaped experimental cage (made from Casanet wire; outer diameter, $21 \mathrm{~cm}$; inner diameter, $10 \mathrm{~cm}$; height, $9.5 \mathrm{~cm}$ ) was located in the middle of the chamber on a metal rack. The cage contained a small feeding dish with a feeder light and, $10 \mathrm{~cm}$ from the feeding dish, a pedestal with a light-interrupting switch. An additional light close to the pedestal was used to provide feedback to the animals during testing. A custom-built feeder mounted at a distance of $120 \mathrm{~cm}$ from the experimental cage was connected to the feeder dish by a flexible tube and dispensed the reward pellets under computer control (Linux workstation, AMD 1900 processor, Tucker-Davis Technologies System II interface with a PA4 attenuator and a PI2 digital $\mathrm{I} / \mathrm{O}$ interface). The computer also controlled the lights, monitored the presence of the mouse on the pedestal via the light-interrupting switch, and controlled the course of events during the experiment, including the presentation of the stimuli. The loudspeaker (Canton Plus XS) broadcasting the sounds was positioned $84 \mathrm{~cm}$ above the pedestal at which the mouse sat during stimulus presentation.
\end{abstract}

\section{Stimulus Generation}

The stimuli were generated with a Linux workstation with a standard sound card (Sound Blaster Model PCI 128, 44.1-kHz sampling rate; Creative Technology) and passed through a programmable attenuator (PA4, Tucker-Davis Technologies). The base sound pressure level of the signal was adjusted by an additional manual attenuator (Hewlett Packard 350D). The stimuli were amplified by a Rotel RMB-1066 amplifier and presented via the Canton XS loudspeaker. Sound pressure levels in the experimental setup were calibrated with a sound level meter (Model 2238 mediator with Model 4188 microphone; Brüel \& Kjær) located at the position where the head of the animal would be during the experiment. The signals used in the experiments were $10-\mathrm{kHz}$ pure tones that had an equivalent duration of $800 \mathrm{msec}$ and cosine rise/fall times of $10 \mathrm{msec}$.

\section{General Procedure and Initial Training}

The general experimental paradigm for both adaptive tracking and the constant-stimuli procedure was a go/no-go procedure reinforcing the subject with food rewards. The experimental protocol was controlled by the workstation using a custom-made program. A trial started when the subject jumped onto the pedestal, and the start was indicated by switching on the pedestal light. After a random waiting interval of between 1 and $7 \mathrm{sec}$, a single test stimulus was presented. The mouse was trained to jump off the pedestal when perceiving a test stimulus (go condition), but otherwise it had to remain on the pedestal. If the subject responded correctly to a test stimulus (i.e., scored a "hit" by leaving the pedestal in the time interval up to $1,300 \mathrm{msec}$ after signal onset), the pedestal light was switched off and a feeder light switched on, a food reward was given, and the next trial started. If the subject missed a test stimulus (i.e., scored a "miss") and remained seated on the pedestal, the next trial was initiated after 1-3 sec (the pedestal light was switched off during this period if the most salient stimulus had been presented in the previous trial). If the subject jumped off the pedestal during the waiting interval before tone onset, the pedestal light was switched off for $1 \mathrm{sec}$ and the trial was restarted with the previous waiting interval. Between $20 \%$ to $30 \%$ of all trials (percentage depending on the procedure used) were catch trials in which no stimulus was given (no-go condition). These trials were used to measure the false alarm rate. If the subject jumped off the pedestal in a catch trial (i.e., scored a false alarm), the pedestal light was switched off for $1 \mathrm{sec}$ and the next trial started. If the subject remained on the pedestal in a catch trial (i.e., scored a "correct rejection"), the pedestal light remained switched on and the next trial started immediately.

Before the initial training, the food-deprived mouse was allowed to explore the experimental cage and retrieve food pellets placed in the feeder cup for a time period of between 20 and $30 \mathrm{~min}$. The initial training used test stimuli that had a sound pressure level of $70 \mathrm{~dB}$, that did not vary from one trial to the next, and that were well above threshold. Catch trials were already used in this training phase to measure the rate of spontaneous responding. The initial waiting interval on the pedestal before stimulus presentation was between 0.1 and $0.2 \mathrm{sec}$. This interval was short enough to allow the mouse to start a stimulus when slowly passing the pedestal. In that phase, leaving the pedestal within $3 \mathrm{sec}$ after stimulus onset was rewarded with a food pellet. When the subject scored several "hits" successively, indicating an at least temporary rise of the hit rate above $50 \%$, the waiting interval was increased (e.g., from 0.2 to $0.5 \mathrm{sec}$ ) and the available response time after stimulus onset was shortened (e.g., to $2 \mathrm{sec}$ ). This procedure continued until the waiting interval ranged between 1 and $7 \mathrm{sec}$ and the mouse jumped reliably within 1 to $1.3 \mathrm{sec}$ after stimulus presentation. During the first few sessions the false alarm rate in catch trials was high, but it decreased over time. The period of initial training lasted between 6 and 8 days, with up to 220 trials a day, until testing commenced with the constant-stimuli procedure described below. In many cases, the mouse needed several days of the initial training in order to learn the task while being presented with relatively short waiting intervals. Thereafter, the waiting interval in the initial training could be lengthened progressively within a relatively short time span. The response time after stimulus onset, however, could be decreased in an even shorter time.

\section{Adaptive-Tracking Procedure}

The adaptive-tracking procedure used in the experiments was the two-down/one-up tracking procedure, resulting in an average response probability of $70.7 \%$ at threshold (Levitt, 1971). At the beginning of each session, the mouse was presented with a stimulus level at least $10 \mathrm{~dB}$ above the estimated hearing threshold (base sound pressure level). When the subject responded with a hit on two consecutive test trials, the sound pressure level of the following stimulus was reduced by $10 \mathrm{~dB}$ (downward tracking; for an example, see Figure 1A). After one miss, the sound pressure level was increased by the same amount (upward tracking). A change in the direction of tracking (either from downward to upward tracking or vice versa) was called a reversal. After the first two reversals during this "warm-up" period, which were not included in the threshold determination, the step size for the reduction and increase in tone level was changed to $5 \mathrm{~dB}$. In order to keep the response under stimulus control, every fifth to eighth trial was a "maintenance trial" in which the tone was presented at a sound pressure level within $1 \mathrm{~dB}$ of the level at the start of tracking. If a subject missed a maintenance trial, the same trial was repeated until a "hit" was recorded. The responses to signals in these trials did not affect the tracking and were not included in the calculation of the threshold. Maintenance trials were introduced when initial observation of the subjects suggested that they were more likely to engage in other behavior not related to the experimental paradigm if no salient stimuli were presented over extended time periods. Introducing salient maintenance trials resulted 

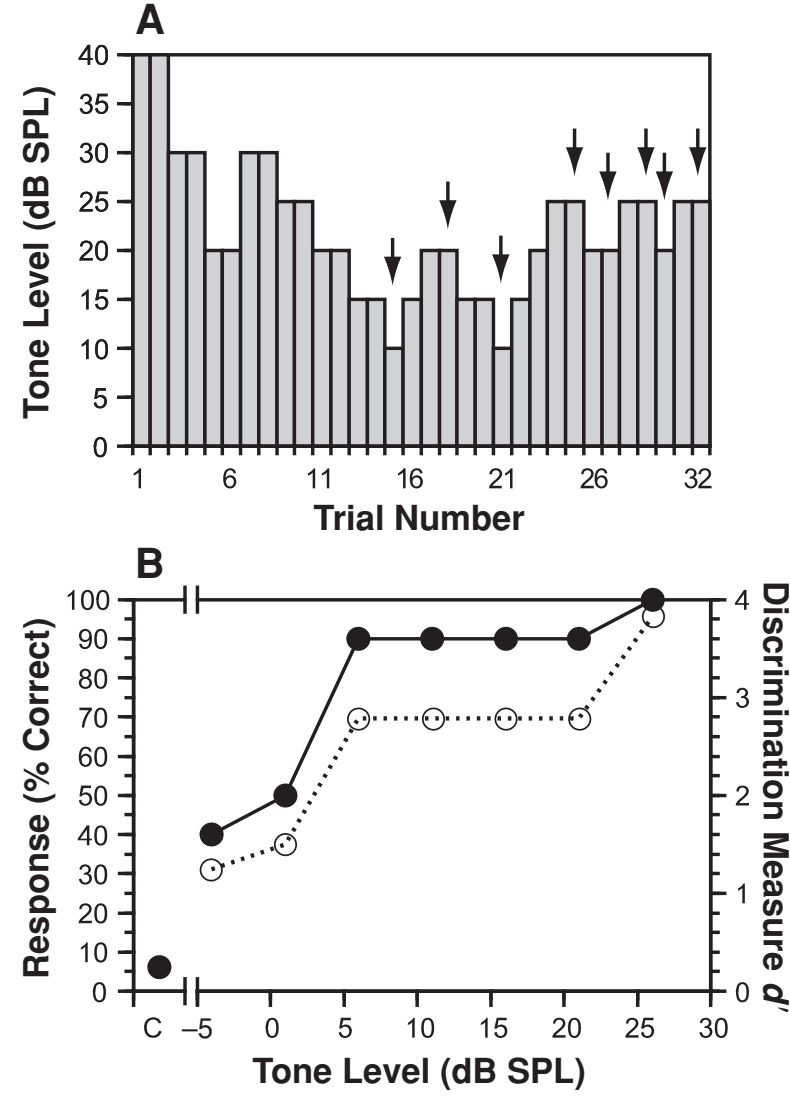

Figure 1. Response to a pure-tone signal as a function of its level. (A) Exemplary data from a session using the adaptive twodown/one-up procedure. Trial Number indicates consecutive trials used in threshold estimation (data are not shown from catch trials $[20 \%]$, used to measure the false alarm rate, and maintenance trials $[12.5 \%-20 \%]$, used to determine the propensity to respond to salient signals). The arrows indicate tone levels at reversal points that could be used for threshold estimation. (B) Exemplary psychometric functions obtained from applying the constant-stimuli procedure (data were obtained 1 day after the session shown in the top graph). The filled circles show response probabilities as a function of the tone level, and the open circles show the corresponding measures of sensitivity (discrimination measure $d^{\prime}$ ). The point labeled $C$ indicates the false alarm rate determined in the catch trials (which were $30 \%$ of all trials).

in a large variation of stimulus levels that is typical of constantstimuli procedures. Catch trials used to determine the subject's false alarm rate were randomly interspersed between tracking trials, with a probability of $20 \%$. A valid session had at least eight reversals (two during the warm-up period and at least six in 5-dB steps during the tracking), no more than $20 \%$ false alarms in catch trials, and a hit rate in maintenance trials of at least $75 \%$. The threshold in the adaptive-tracking procedure was calculated as the average value of the signal levels of the first six reversals following the warm-up period (see the first six arrows in Figure 1A). If during tracking for the threshold the subject's responses would result in the tone's sound pressure level exceeding the base sound pressure level, the session was discarded, and no threshold was determined.

\section{Constant-Stimuli Procedure}

In the constant-stimuli procedure, a block of 10 trials, consisting of a set of 7 test trials in which the sound pressure level of the tone differed in steps of $5 \mathrm{~dB}$ (covering a range from 0-30 dB below the base sound pressure level) and 3 catch trials, was repeated 11 times, resulting in a total session length of 110 trials. The sound pressure level of the test tones was generally chosen in such a way that the two signals with the lowest levels were below threshold and the two signals with the highest levels were well above threshold. The trials within each block were presented in random order, thus making it impossible for the mouse to anticipate the next stimulus. The first block of 10 trials served as a warm-up period. At the end of a session, a psychometric function was compiled summarizing the results of the 100 trials, after excluding the trials from the warm-up period. This psychometric function (see Figure 1B) either showed the probability of correct response upon presentation of a tone or the measure of sensitivity $d^{\prime}$ (which can be calculated from the probability of correct response and the false alarm rate; see Green \& Swets, 1966) in relation to the sound pressure level of the stimulus. Sessions were excluded from the analysis if the percentage of false alarms was greater than $20 \%$ or if the average hit rate in the trials with the two most salient test stimuli (i.e., those with the highest sound pressure level) was less than $75 \%$.

The tone detection threshold was calculated in different ways by linear interpolation between the measured response at the sound pressure level of the two stimuli (differing in level by $5 \mathrm{~dB}$ ) that were just above and below the estimated threshold. First, the same threshold criterion was applied as in the adaptive-tracking procedure $(70.7 \%$ correct responses). Second, a threshold was defined as the sound pressure level that resulted in $50 \%$ correct responses. Third, thresholds were calculated using signal detection theory and threshold criteria of $d^{\prime}=1.0$ and 1.8 , respectively. To evaluate the effect of the session length on the threshold estimate, thresholds for $d^{\prime}=1.8$ were also calculated for sessions with a length of either 70 or 50 trials following the warm-up period. These data were obtained by truncating the 110-trial sessions and then repeating the analysis described above (i.e., the shortened sessions had to meet all criteria for inclusion in the analysis).

\section{RESULTS}

For 5 of the 6 animals tested, tone detection thresholds could be obtained for both the adaptive-tracking procedure and the constant-stimuli procedure. One female was excluded from further experiments, and the few data obtained from that subject are not shown below, because the subject stopped responding to the sounds in the initial phase of the experiment.

Figure 2 gives an example of average psychometric functions from female w96; these graphs were constructed by summarizing data that met the criteria for analysis from 11 sessions of adaptive tracking and 18 sessions of the constant-stimuli procedure. As is obvious from Figure 2A, data from the psychometric function obtained with the adaptive-tracking procedure are concentrated on the part of the function representing signal levels that generally result in a high response probability. During downward tracking, the subject mostly operates above a response rate of $70.7 \%$; when the rate drops below the threshold of $70.7 \%$, a reversal to upward tracking results in an increased sound pressure level and in a corresponding increase in response rate. The discrimination measure $d^{\prime}$ for the signals presented during tracking generally has a value of 1.8 or higher (Figure 2A). In the constant-stimuli procedure, however, the psychometric function spans a range of response probabilities from about 20\%-90\% (Figure 2B); that is, it ranges from a value close to the false alarm rate 

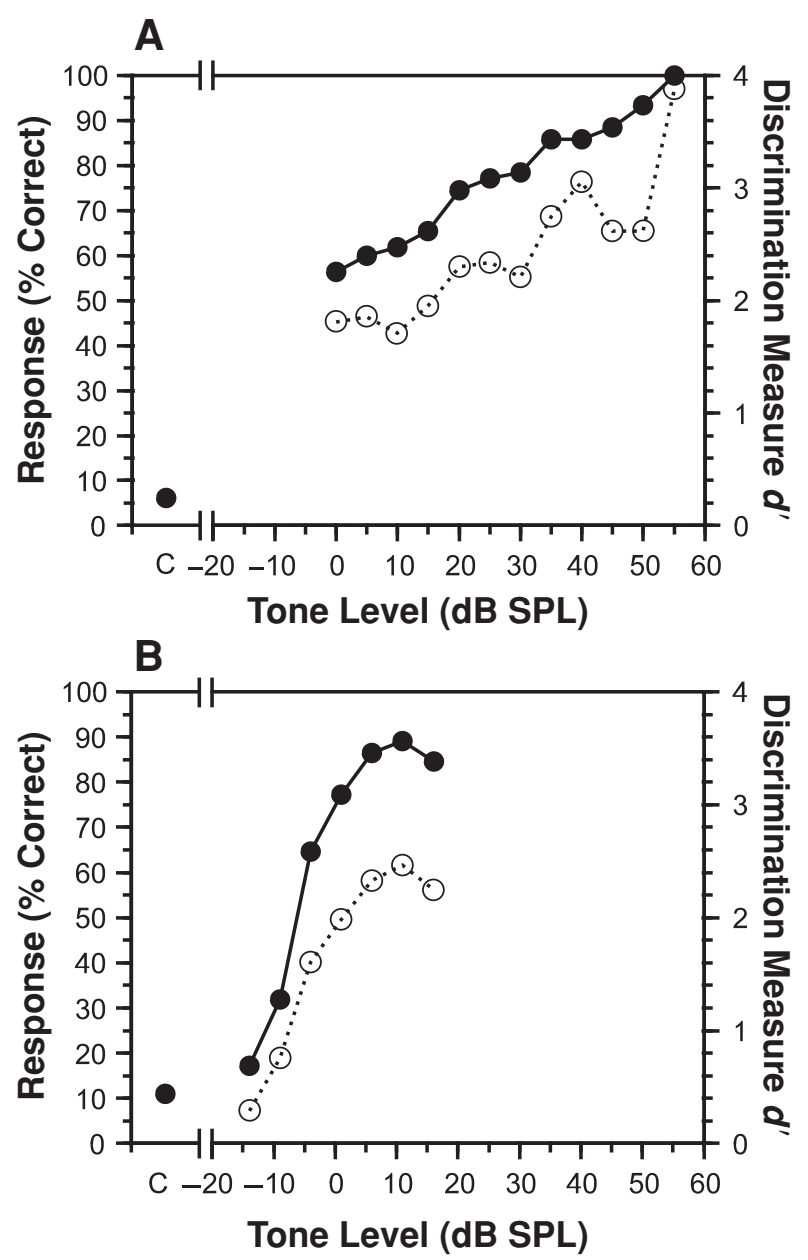

Figure 2. Average psychometric functions of 1 subject derived from (A) sessions applying the adaptive-tracking procedure $(n=$ 18 sessions with six reversals; only data for stimulus levels presented at least five times are shown) and (B) sessions applying the constant-stimuli procedure $(n=11$ sessions with identical attenuator settings). In the graphs, filled circles show response probabilities as a function of level of the test tone, and open circles show the corresponding measures of sensitivity (discrimination measure $d^{\prime}$ ). The point labeled $C$ shows the average false alarm rate of the subject in the specific procedure.

(11\%) to a value near $100 \%$. The corresponding values of the discrimination measure $d^{\prime}$ range from 0.3 to 2.5 (Figure 2B). In this example, it is obvious that similar response probabilities of $70.7 \%$ are observed at a sound pressure level of the signal that is $19.5 \mathrm{~dB}$ higher in the adaptivetracking procedure than in the constant-stimuli procedure (i.e., at tone levels of $17.9 \mathrm{~dB}$ SPL in the adaptive-tracking procedure and $-1.6 \mathrm{~dB}$ SPL in the constant-stimuli procedure). This finding can also be demonstrated by analyzing data from single sessions. Figure 1 shows data from two sessions that were conducted with the same animal on two consecutive days. In the constant-stimuli procedure, a response rate of $70.7 \%$ was observed for tones at a sound pressure level of about $4 \mathrm{~dB}$ SPL. In the adaptivetracking procedure, however, the animal's threshold first varied around $15 \mathrm{~dB}$ SPL and then around 20-25 dB SPL when tracking the threshold based on the $70.7 \%$ response probability inherent in the two-down/one-up rule. In the sessions shown in Figure 1, the mouse subject obtained about the same average amount of reinforcements per trial in both procedures ( 28 reinforcements in the adaptivetracking session of 32 tracking trials, 5 maintenance trials, and 8 catch trials, or 0.62 reinforcements per trial; 59 reinforcements in the constant-stimuli session of 70 test trials, 7 warm-up trials, and 33 catch trials, or 0.54 reinforcements per trial).

The difference in the thresholds obtained with the two different procedures is evident in all subjects that were tested (Figure 3). Thresholds obtained with the constantstimuli procedure were significantly lower than those obtained with the adaptive-tracking procedure (repeated measures ANOVA, $p=.006$ ). For the 5 subjects, the thresholds obtained with the constant-stimuli procedure at a session length of 100 trials ranged on average between -15 and $4 \mathrm{~dB}$ SPL, whereas the thresholds obtained with the adaptive-tracking procedure for the same threshold criterion ranged on average between 0 and $34 \mathrm{~dB}$ SPL. Not only were the thresholds higher in the adaptivetracking procedure, but also variation in the threshold appears to have increased in the adaptive-tracking procedure in comparison with the constant-stimuli procedure (there was a trend for the standard deviation of the threshold to be smaller in the constant-stimuli procedure than in the adaptive-tracking procedure according to a repeated measures ANOVA, $p=.07)$. Standard deviations varied between 1 and $6 \mathrm{~dB}$ for the constant-stimuli procedure and 3 and $16 \mathrm{~dB}$ for the adaptive-tracking procedure.

In psychophysical studies of auditory sensitivity, different threshold criteria have been applied. In go/no-go procedures, a threshold criterion of $50 \%$ responses upon presentation of a stimulus is commonly used, with some constraints on the false alarm rate in the experiment (see, e.g., Dooling \& Okanoya, 1995; May, Huang, Aleszczyk, \& Hienz, 1995). In human psychophysical studies and in some studies of animal psychophysics, thresholds are estimated by using signal detection theory and applying a threshold criterion of $d^{\prime}=1.0$ or $d^{\prime}=1.8$ (e.g., Dooling \& Okanoya, 1995; Green \& Swets, 1966). We compared the thresholds obtained when these different criteria were applied in the constant-stimuli procedure and found that the thresholds depended on the criterion (repeated measures ANOVA, $p<.001$; see Figure 4). Threshold values were not different if the criteria $70.7 \%$ correct and $d^{\prime}=$ 1.8 were applied (Tukey test, $p=.47$ ) or if the criteria $50 \%$ correct and $d^{\prime}=1.0$ were applied (Tukey test, $p=$ $.73)$. The threshold values obtained with the more conservative response criteria $\left(70.7 \%\right.$ correct and $\left.d^{\prime}=1.8\right)$ were 3.1 to $5.7 \mathrm{~dB}$ higher than those obtained with the less conservative criteria (50\% correct and $d^{\prime}=1.0$; Tukey test, all $p$ s $\leq .05$ ).

When applying the constant-stimuli procedure, the length of the session could be reduced from 100 to 50 trials (following the 10 warm-up trials in each session) without a significant effect on the threshold and its variability 


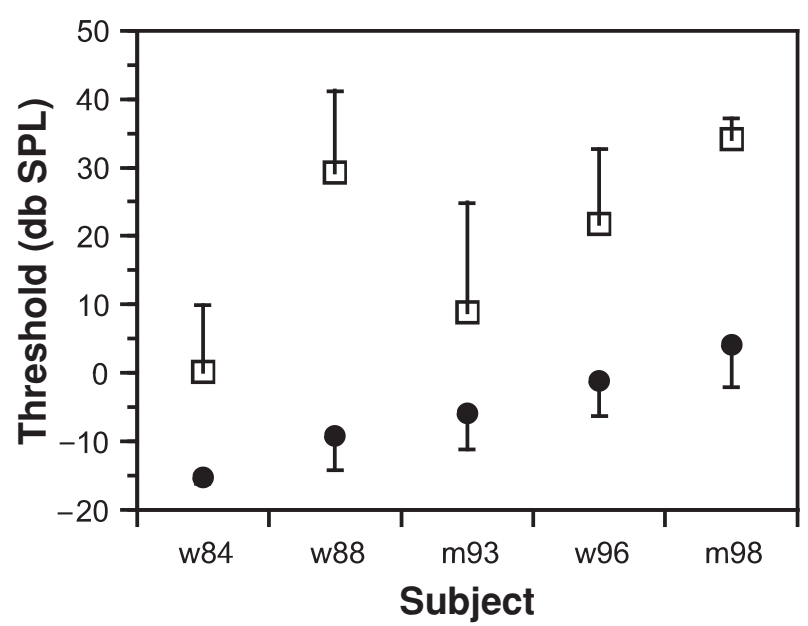

Figure 3. Average thresholds and associated standard deviations for detection of a tone by 5 subjects. The open squares indicate thresholds obtained from an analysis of six reversals in the adaptive-tracking procedure. The filled circles indicate thresholds obtained with the constant-stimuli procedure for sessions in which 100 trials were analyzed. The threshold criterion for both the adaptive-tracking and constant-stimuli procedures was $\mathbf{7 0 . 7 \%}$ correct responses upon presentation of a signal.

(Figure 5). There was only a minute threshold increase of $0.3 \mathrm{~dB}$ if the warm-up trials were omitted, and in that case the standard deviation was only slightly increased by $0.3 \mathrm{~dB}$ (none of the differences was significant according to a $t$ test, $p>.8)$. The shortest session length in the constant-stimuli procedure, 50 trials, comes close to the average session length of 47 trials ( \pm 10 trials $S D$ ) in the adaptive-tracking procedure up to the point of six reversals.

\section{DISCUSSION}

Absolute tone detection thresholds determined in psychoacoustic experiments with the same NMRI mouse subjects and an identical response criterion were on average $24 \mathrm{~dB}$ lower when using a constant-stimuli procedure than the thresholds obtained with a two-down/one-up adaptive-tracking procedure. Furthermore, the standard deviations of thresholds determined with the adaptivetracking procedure were on average about double those with the constant-stimuli procedure. Thus, in the NMRI mouse, the constant-stimuli procedure appears to be better suited to determine auditory sensory thresholds than is the adaptive-tracking procedure. One possible explanation for this difference could be that the subjects were inattentive or performed other, task-unrelated behaviors (e.g., grooming) that temporarily resulted in a reduced response probability. It is well known from human psychoacoustic studies that inattentiveness may pose a problem in the estimation of sensory thresholds (see, e.g., Green, 1995; Leek, Hanna, \& Marshall, 1991). A reduction of response probability in the adaptive-tracking procedure shifts the working point of the subjects to a higher sound pressure level when they perform. In the constant-stimuli procedure, a temporarily reduced response probability makes the psychometric function more shallow but does not have such a large effect on the threshold value as it does in the adaptivetracking procedure, since in the constant-stimuli procedure the tone levels and their distribution are preselected and do not vary over a large range based on the subject's responses. It is conceivable that the mice tracked higher sound levels to obtain rewards with a lower attentive load.

Considerable variation exists in the mean values of auditory threshold data and standard deviations for various strains of the house mouse using operant-conditioning procedures. Table 1 compares the data of different studies from a frequency range of $8-10 \mathrm{kHz}$. Part of the variation may be explained by strain differences. However, the studies also differ in the acoustic parameters of the stimuli (e.g., duration, ramps, and repeats may affect the threshold), the method of stimulus presentation, and the threshold criterion. In our data, the threshold criterion did result in a variation of threshold by $6 \mathrm{~dB}$ in the constantstimuli procedure. Tracking procedures in general appear to result in less sensitive thresholds in the mouse than do constant-stimuli procedures. However, more studies are needed that compare results obtained in close temporal succession with the same apparatus and subjects before a final conclusion can be drawn about the factors underlying the threshold differences determined using different operant procedures. Furthermore, there may also be differences in the responses of various mouse strains to the different procedures.

In addition, sensitive auditory thresholds have been determined with classical conditioning procedures (e.g., Markl \& Ehret, 1973, found thresholds in the NMRI mouse via classical conditioning that were in the range of threshold values reported in Table 1 for operant conditioning). Thresholds have also been determined using

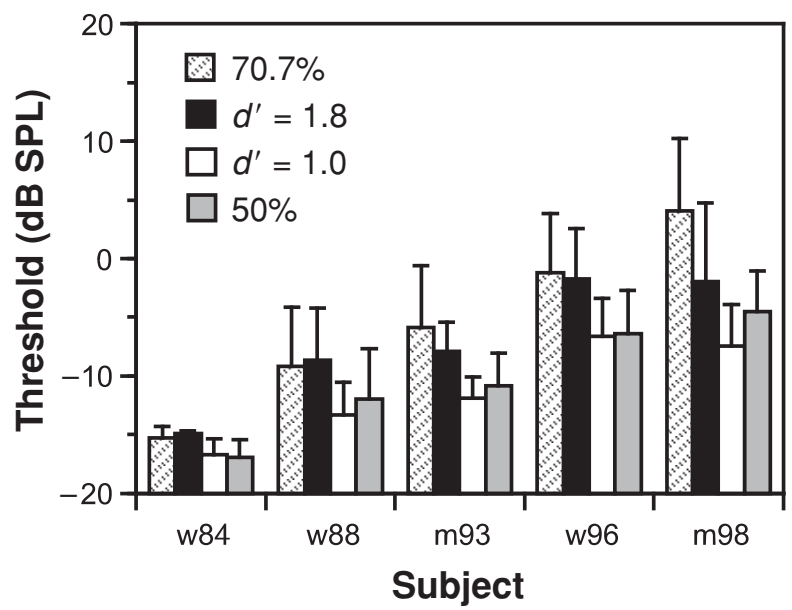

Figure 4. Average thresholds and associated standard deviations for detection of a tone by 5 subjects with the constant-stimuli procedure, as a function of threshold criterion. Sessions consisted of 110 trials, but only the last $\mathbf{1 0 0}$ trials were used for threshold estimation; the first 10 trials were discarded as warm-ups. 


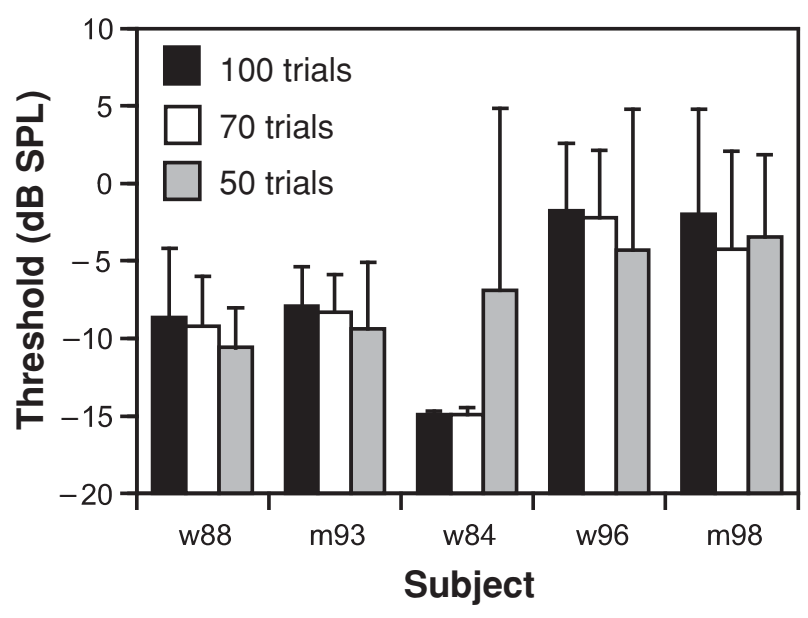

Figure 5. Average thresholds and associated standard deviations for detection of a tone by 5 subjects with the constant-stimuli procedure, as a function of length of the session (i.e., the number of trials used for threshold estimation, not including 10 warm-up trials). The threshold criterion for all trials was $d^{\prime}=1.8$.

acoustic startle responses such as the Preyer reflex (see, e.g., Schleidt, 1952) or the galvanic skin response (e.g., Berlin, 1963). These physiological responses, however, are only elicited by a stimulus with a high level, and therefore result in considerably higher thresholds than those obtained with operant procedures (for such a comparison of results, see Markl \& Ehret, 1973). However, unlike these reflexes themselves, the inhibition of acoustic startle responses by sound pulses preceding the startle stimulus (prepulse inhibition, or PPI) can also be used to determine sensitive auditory thresholds. In mice, only masked threshold values have been measured so far using PPI (Ison \& Agrawal, 1998), and these were slightly higher than would be expected on the basis of known values of the critical bandwidth of the mouse (Ehret, 1976). No absolute thresholds have been measured in the mouse using PPI, but this method has been shown to be suitable in rats and guinea pigs for determining absolute thresholds that are as sensitive as those derived from auditory brain stem responses (Young \& Fechter, 1983).

In psychoacoustics, adaptive-tracking procedures have been considered to be more efficient psychophysical procedures than constant-stimuli procedures, since most data are collected close to the final threshold (see, e.g., Niemiec \& Moody, 1995). Here we have shown that in the NMRI mouse, the session length in the constant-stimuli procedure can be reduced without a large loss of accuracy, in which case the constant-stimuli procedure becomes nearly as effective (based on the number of trials) as the two-down/one-up adaptive-tracking procedures. A pretest is necessary, however, in order to find suitable stimulus values for presentation in the constant-stimuli procedure. A constant-stimuli procedure with a food reward as reinforcement is also a suitable method for determining other sensory capabilities of the mouse (e.g., the perception of stimulus duration; Klink \& Klump, 2004).

To make adaptive-tracking procedures more suitable for determining sensory thresholds in the NMRI mouse, one would have to find ways to cope with temporary lapses in participation in the experiments. Methods to cope with inattentiveness have been developed for the study of human psychoacoustics, and these may possibly be applied in order to develop suitable adaptive-tracking procedures for the NMRI mouse (see, e.g., Leek et al., 1991). The result that the constant-stimuli procedure can lead to lower and more stable thresholds than does the adaptive-tracking procedures, even with the same threshold criteria, may not apply only to studies of auditory sensitivity in the mouse, but may be applicable to the study of a range of sensory modalities in the mouse and other animals.

\section{REFERENCES}

BerLin, C. I. (1963). Hearing in mice via GSR audiometry. Journal of Speech \& Hearing Research, 13, 359-368.

Birch, L. M., Warfield, D., Ruben, R. J., \& Mikaelian, D. O. (1968). Behavioral measurements of pure tone thresholds in normal CBA-J mice. Journal of Auditory Research, 8, 459-468.

DAI, H. (1995). On measuring psychometric functions: A comparison of the constant-stimulus and adaptive up-down methods. Journal of the Acoustical Society of America, 98, 3135-3145.

Table 1

Thresholds Determined in Various Strains of the House Mouse Using Operant-Conditioning Procedures

\begin{tabular}{|c|c|c|c|c|c|}
\hline Strain & $N$ & Stimulus & $\begin{array}{l}\text { Threshold } \\
\text { (dB SPL) }\end{array}$ & $S D$ & Reference \\
\hline \multicolumn{6}{|c|}{ Constant-Stimuli Procedure } \\
\hline NMRI & 5 & single $800-\mathrm{msec}$ tone, $10 \mathrm{kHz}$ & -6 & 5 & The present study \\
\hline $\mathrm{C} 57 \mathrm{BL} / 6$ & 3 & single $800-\mathrm{msec}$ tone, $10 \mathrm{kHz}$ & 8 & 1 & Weik, 2004 \\
\hline $\mathrm{CBA} / \mathrm{J}$ & 3 & 3 -sec series of $250-\mathrm{msec}$ tones, $8 \mathrm{kHz}$ & 23 & - & Prosen, Dore, \& May, 2003 \\
\hline Wild mice & 3 & single 10 -sec tone, $8 \mathrm{kHz}$ & 6 & 2 & Heffner \& Masterton, 1980 \\
\hline \multicolumn{6}{|c|}{ Two-Down/One-Up Adaptive-Tracking Procedure } \\
\hline NMRI & 5 & single $800-\mathrm{msec}$ tone, $10 \mathrm{kHz}$ & 19 & 10 & The present study \\
\hline \multicolumn{6}{|c|}{ Method of Limits (Unidirectional Adaptive-Tracking Procedure) } \\
\hline NMRI & 4 & series of five 3 -sec tones, $10 \mathrm{kHz}$ & 13 & 1 & Markl \& Ehret, 1973 \\
\hline $\mathrm{C} 57 \mathrm{BL} / 6$ & 17 & 10 -sec series of 150 -msec tones, $9.8 \mathrm{kHz}$ & 32 & 12 & Mikaelian, Warfield, \& Norris, 1974 \\
\hline $\mathrm{CBA} / \mathrm{J}$ & 10 & 10 -sec series of 150 -msec tones, $9.8 \mathrm{kHz}$ & 29 & 21 & Birch, Warfield, Ruben, \& Mikaelian, 1968 \\
\hline
\end{tabular}

Note-All studies above except that of Heffner and Masterton (1980; conditioned avoidance) have been performed with the go/no-go procedure. 
Dooling, R. J., \& OKanoya, K. (1995). The method of constant stimuli in testing auditory sensitivity in small birds. In G. M. Klump, R. J. Dooling, R. R. Fay, \& W. C. Stebbins (Eds.), Methods in comparative psychoacoustics (pp. 161-169). Basel: Birkhäuser.

EHRET, G. (1976). Critical bands and filter characteristics in the ear of the housemouse (Mus musculus). Biological Cybernetics, 24, 35-42.

Green, D. M. (1995). Maximum-likelihood procedures and the inattentive observer. Journal of the Acoustical Society of America, 97 3749-3760.

Green, D. M., \& Swets, J. A. (1966). Signal detection theory and psychophysics. New York: Wiley.

HeFFner, H. E., \& HeFfNer, R. S. (2001). Behavioral assessment of hearing in mice. In J. F. Willott (Ed.), Handbook of mouse auditory research (pp. 19-29). Boca Raton, FL: CRC Press.

Heffner, H. [E.], \& Masterton, B. (1980). Hearing in glires: Domestic rabbit, cotton rat, feral house mouse, and kangaroo rat. Journal of the Acoustical Society of America, 68, 1584-1599.

Ison, J. R., \& Agrawal, P. (1998). The effect of spatial separation of signal and noise on masking in the free field as a function of signal frequency and age in the mouse. Journal of the Acoustical Society of America, 104, 1689-1695.

Klink, K. B., \& Klump, G. M. (2004). Duration discrimination in the mouse (Mus musculus). Journal of Comparative Physiology A, 190, 1039-1046.

LEeK, M. R. (2001). Adaptive procedures in psychophysical research. Perception \& Psychophysics, 63, 1279-1292.

Leek, M. R., Hanna, T. E., \& Marshall, L. (1991). An interleaved tracking procedure to monitor unstable psychometric functions. Journal of the Acoustical Society of America, 90, 1385-1397.

LeeK, M. R., Hanna, T. E., \& Marshall, L. (1992). Estimation of psychometric functions from adaptive tracking procedures. Perception \& Psychophysics, 51, 247-256.

LevitT, H. (1971). Transformed up-down methods in psychoacoustics. Journal of the Acoustical Society of America, 49, 467-477.

Markl, H., \& Ehret, G. (1973). Die Hörschwelle der Maus (Mus musculus): Eine kritische Wertung der Methoden zur Bestimmung der Hörschwelle eines Säugetiers. Zeitschrift für Tierpsychologie, 33, 274-286.

May, B. J., Huang, A. Y., AleszczyK, C. M., \& Hienz, R. D. (1995).
Design and conduct of sensory experiments for domestic cats. In G. M. Klump, R. J. Dooling, R. R. Fay, \& W. C. Stebbins (Eds.), Methods in comparative psychoacoustics (pp. 95-108). Basel: Birkhäuser.

Mikaelian, D. O., Warfield, D., \& NorRis, O. (1974). Genetic progressive hearing loss in the $\mathrm{C} 57 / \mathrm{b} 16$ mouse. Acta Otolaryngologica, 77, 327-334

Niemiec, A. J., \& Moody, D. B. (1995). Constant stimulus and tracking procedures for measuring sensitivity. In G. M. Klump, R. J. Dooling, R. R. Fay, \& W. C. Stebbins (Eds.), Methods in comparative psychoacoustics (pp. 65-77). Basel: Birkhäuser.

Prosen, C. A., Bath, K. G., Vetter, D. E., \& May, B. J. (2000). Behavioral assessments of auditory sensitivity in transgenic mice. Journal of Neuroscience Methods, 97, 59-67.

Prosen, C. A., Dore, D. J., \& May, B. J. (2003). The functional age of hearing loss in a mouse model of presbycusis: I. Behavioral assessments. Hearing Research, 183, 44-56.

SchleIdT, W. M. (1952). Reaktionen auf Töne hoher Frequenz bei Nagern. Naturwissenschaften, 39, 69-70.

Waterston, R. H., Lindblad-Toh, K., Birney, E., Rogers, J., Abril, J. F., Agarwal, P., ET AL. (2002). Initial sequencing and comparative analysis of the mouse genome. Nature, 420, 520-562.

Watson, A. B., \& FitZhugh, A. (1990). The method of constant stimuli is inefficient. Perception \& Psychophysics, 47, 87-91.

WEIK, V.-N. (2004). Untersuchungen zu Maskierungseffekten innerhalb und zwischen auditorischen Filtern bei der Hausmaus (Mus musculus). Unpublished diploma thesis, Oldenburg University, Oldenburg, Germany.

Willott, J. F., Tanner, L., O'Steen, J., Johnson, K. R., Bogue, M. A., \& GAGNON, L. (2003). Acoustic startle and prepulse inhibition in 40 inbred strains of mice. Behavioral Neuroscience, 117, 716-727.

Young, J. S., \& FECHTER, L. D. (1983). Reflex inhibition procedures for animal audiometry: A technique for assessing ototoxicity. Journal of the Acoustical Society of America, 73, 1686-1693.

Zheng, Q. Y., Johnson, K. R., \& ERWAY, L. C. (1999). Assessment of hearing in 80 inbred strains of mice by ABR threshold analyses. Hearing Research, 130, 94-107.

(Manuscript received August 10, 2004; revision accepted for publication January 3, 2005.) 